\title{
The Use of Umbilical Stem Cells
}

\author{
Peter Cassar, Renald Blundell \\ University of Malta, Msida, Malta \\ Email: peterkcassar@gmail.com
}

Received 26 October 2015; accepted 24 January 2016; published 28 January 2016

Copyright (C) 2016 by authors and Scientific Research Publishing Inc.

This work is licensed under the Creative Commons Attribution International License (CC BY).

http://creativecommons.org/licenses/by/4.0/

c) (7) Open Access

\begin{abstract}
The use of umbilical stem cells in tissue engineering is gaining in popularity. Some of the various uses of these umbilical stem cells are highlighted in this review, focusing mainly on their cartilage, bone and neuronal differentiating abilities. The review will also shed light on the application of these abilities in human clinical trials to repair, protect and treat diseases, e.g. neurodegenerative diseases such as Alzheimer's and cerebral palsy. A side by side comparison with bone marrow mesenchymal stem cells (current gold standard for tissue engineering) will give a better idea of the viability and efficiency of umbilical stem cell use.
\end{abstract}

\section{Keywords}

HUCMSCs, Bone, Cartilage, Neuronal

\section{Introduction}

\subsection{Anatomy of Umbilical Cord}

The human umbilical cord (UC) in itself contains distinct anatomical regions consisting of an umbilical vein, two umbilical arteries, cord lining, and Wharton's jelly (this jelly-like tissue surrounds the blood vessels and plays the functional role in supporting the vessels) [1].

\subsection{Uses of Umbilical Stem Cells}

Throughout recent history the literature has described each of these regions as giving rise to a great number of fibroblastoid mesenchymal stem cells (MSCs). Refer to Figure $\mathbf{1}$ for a simple illustration of cord anatomy and differentiating abilities of umbilical stem cells. Human umbilical cord derived MSCs (HUCMSCs) isolated from the cord's structures; arteries, vein, cord lining and Wharton's jelly have all been shown to be multipotent in nature, differentiating into many cell types such as adipocytes [1], osteoblasts [2], hepatocytes [3], chondrocytes 


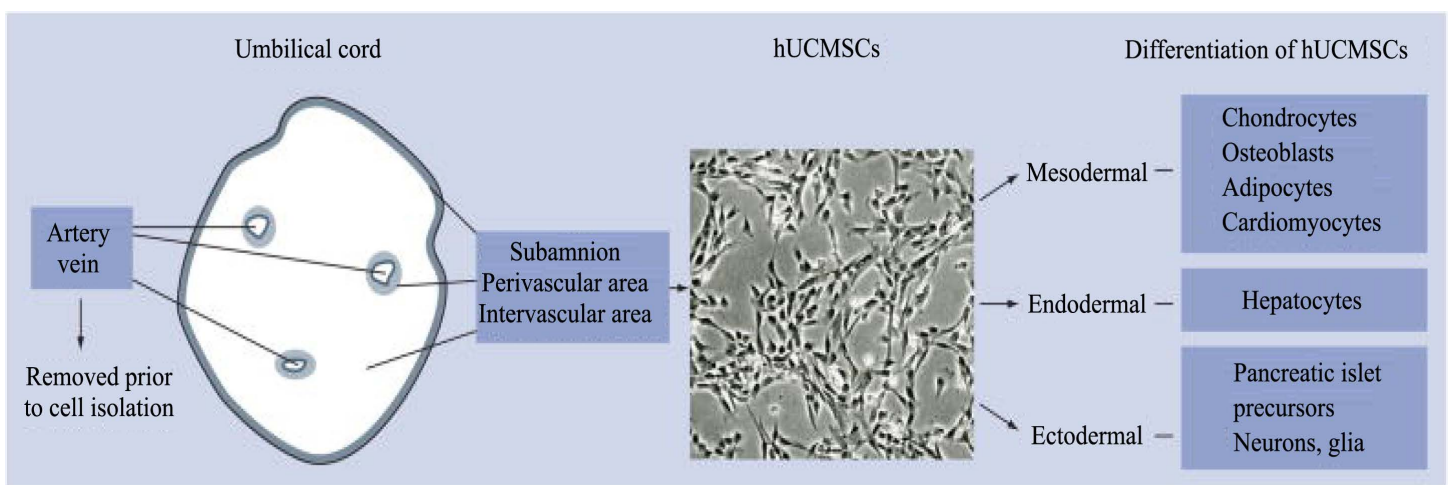

Figure 1. Diagrammatic representation of cord cross-section and differentiation schematic diagram of HUCMSCs. This figure provides a simple diagrammatic representation of the umbilical cord with its two arteries and vein separated by an intervascular area which is immediately surrounded by a perivascular area and subamniotic later after that. As listed above these mesenchymal stromal cells are capable in differentiating into many different cells types and tissues. These stem cells' vast differentiating ability coupled with their natural abundance leads to them being a very reasonable and efficient source for tissue engineering [2].

[2], haemopoetic cells [4], cardiac [5] and neural cells [6] [7]. Umbilical cord stem cells have also been proved promising in possible treatment of several diseases and conditions such as diabetes [8], certain diabetic wounds [9], and brain damage associated with neonatal hypoxia [10], stroke [11], autism [12], acute liver failure [13], cerebral palsy [6] [14] and Alzheimer's [15]. They have also been found to express markers typically expressed by bone marrow derived MSCs (BMSCs), whilst testing negative for markers of haematopoietic and macrophage nature [1].

\subsection{Umbilical Stem Cells VS Bone Marrow Stem Cells}

For a long time bone marrow has been considered as the "crème de la crème" when it comes to the derivation of MSCs and their use in human stem cell engineering has become somewhat of a standard. However, bone marrow extraction comes with ethical issues and various problems related to painful harvesting and donor site injury and morbidity. However, UCs are being looked into as a potential source of MSCs for various advantageous reasons; not only do they proliferate rapidly in culture and are thought to be immune privileged against certain antigens but they are considered as waste within medical community and therefore their potential use in research has little ethical concern unlike bone marrow harvesting [1].

BMSCs in comparison with HUCMSCs have shown notable changes with increasing patient age, such as lower differentiation potential in vitro, reduction in available cell number and also longer doubling times. As mentioned, HUCSMs are able to tolerate, to a certain degree, the introduction of antigens without elicting an automatic inflammatory immune response in vitro, making them worthy candidates for allogenic based therapies. Thus UC MSCs are proving to be an appropriate source of primitive MSCs that can be cryogenically stored in banks and thawed when their therapeutic abilities are needed [1].

\subsection{This Review}

This review will serve to identify some of the various uses of human umbilical cord stem cells. Throughout the review the advantages as well as shortcomings of this MSC type will be highlighted, also giving an insight into the future of HUCMSCs.

\section{Cartilage: HUCMSCs Promising Finds in Fibrocartilage Engineering. Possibilities for Hyaline Cartilage in the Future}

\subsection{Growth Factors and Chondrocyte Differentiation}

Damaged cartilage has minimal self-regeneration abilities. Compared with the present day treatments such as artificial prosthesis, cell-based implantation and microfracture, stem cells have the potential of becoming the go to treatment in terms of cartilage function restoration. Due to the self-generating and differentiating abilities of 
mesenchymal stem cells such as HUMSCs, stem cells have become a viable source for cartilage engineering. Tissue growth factor (TGF) isoforms (TGF- $\beta 1, \beta 2$ and $\beta 3$ ) have been found to trigger the differentiation of MSCs into chondrocytes while dexamethasone has been found to improve and enhance differentiation [2]. Insulin-like growth factor-1 (IGF-1) and its role in chondrogenic differentiation still remains somewhat controversial. After the removing insulin from the insulin-transferrin-selenious acid Premix (1X serum substitute Premix), a serum-free chondrogenic foetal bovine serum artificial medium, IGF-I showed equal chondroinductive capability with MSCs when compared with TGF- $\beta$ isoforms. Experimentation also showed that a chondrogenic anabolic medium with IGF-1, previously containing TGF-B instead of IGF-1, increased noticeably the synthesis and gene expression of collagen type II apart from increasing collagen production in relation to chondrogenic medium [16].

TGF- $\beta$ s, as with other mesenchymal stem cells, are able to influence HUCMSCs and direct them towards a chondrogenic lineage. They are grown in 3D cell pellets and specially designed polyglycolic acid (PGA) scaffolds (this biodegradable polyester, PGA, is being exploited in a variety of medical applications). However in more recent years a new method of HUCMSC chondrocyte differentiation was developed involving the upregulation of SOX-9, a main regulatory gene in chondrogenesis and the initiation of the chondrogenic differentiation of HUCMSCs at the level of mRNA [17].

\subsection{Inconsistent Results}

Confirmation of chondrogenic differentiation in terms of extracellular proteins was verified by immunohistochemal staining and antigen retrieval for type II collagen and also by Alcian blue staining for glycosaminoglycans (GAGs) [2]. However studies have yielded inconsistent results regarding HUCMSCs chondrogenic differentiation.

In the control group (not containing TGF $\beta 1$ ) of an experiment conducted by [18], there was a noted positive staining for both collagen type II and GAGs. However, in an experiment of a similar nature, there was no type II collagen production noted within the control group, although in HBMSCs only a slight amount of type II collagen was detected [19], contrasting significantly with previous HBMSC studies where the formation of collagen type had been abundant [2].

\subsection{HUCMSCs Outcompeting HBMSCs and Possible Applications}

In this study both HBMSCs and HUCMSCs contributed to the formation of collagen type 1 . However the HUCMSC group produced larger pellets, with a filamentous extracellular matrix bettering that produced by HBMSCs. Furthermore, the glycosaminoglycan (GAG) quantification showed that a subpopulation of the HUCMSCs, known as the perivascular cells, produced an amount of GAGs similar to that produced by HBMSCs after just 21 days, admittingly the first week saw the perivascular cells producing less GAGs than then the HBMSC groups. Comparing HBMSCs with HUCMSCs the latter has better differentiating ability, can generate both type I and II collagen (as found in both biomaterial-based and cell pellet culture). HUCMSCs also outcompeted HBMSCs with better extracellular matrix synthesis and increased production of collagen and GAGs. However HBMSCs was found to advance more along the chondrogenic lineages with increased protein levels and increased levels of collagen type II gene expression. This study continued to secure HUCMSCs' place in fibrocartilage tissue engineering, being an appropriate substitute for the development of fibrocartilagenous tissues. Possible applications include the treatment of damaged intervertebral discs, temporomandibular joint (TMJ) discs, and knee menisci [2].

\subsection{HUCMSCs Outperform TMJ Condylar Cartilage Cells}

The very first in depth study into in vitro utilization of HUCMSCs cartilage differentiating abilities, compared the TMJ regeneration between the stem cells and TMJ condylar cartilage cells. After both types of cells were subjected to a 4-week long culture in PGA scaffolds, not only did the HUCMSCs match the native TMJ cartilage in terms of the similar fibrocartilage-like tissue produced but actually performed better than the TMJ condylar cartilage cells, with an increase of both collagen and GAGs as well as cellularity. Due to the limited supply of TMJ cells compared to the more abundant HUCMSCs, these MSCs were suggested as an ideal source 
for TMJ condylar cartilage regeneration [2].

\subsection{Optimum Seeding Cell Density}

After having established that HUCMSCs indeed were viable for fibrocartilage tissue engineering, further studies were undertaken in order to discover what were the effects of cell seeding densities on different criteria such as matrix synthesis, cell proliferation and their differentiation. After experimenting with different cell densities, a seeding density higher than that of 25 million cells/ml was advised for future studies in fibrocartilage tissue engineering. The HUCMSCs differentiated along a fibrocartilage lineage with excellent results, producing a very viable fibrocartilage-like tissue with the necessary co-existing components of aggrecan, collagen type I and II. These studies proved HUCMSCs, yet again, to be an ideal cell alternative for fibrocartilage regeneration [20].

\subsection{Future of HUCMSCs in Hyaline Cartilage Engineering}

The use of HUCMSCs in the engineering of hyaline cartilage is still an undergoing study. Some of the main issues lie in the need for further investigation into the required signals that will further enhance and boost type II collagen production necessary for hyaline cartilage engineering [21]. Recent studies have hinted towards the possible use of hypertrophy markers in studies to come, in order to characterize the phenotype of the developed chondrocytes more accurately. Also inhibiting terminal differentiation may not always lead to stable hyaline cartilage, as the correct balance of signals must be created from the very start of culture onwards [22]. However hyaline engineering using HUCMSCs is still quite young. Maybe exploring the influence of factors such as coculture, chemical signals such as aggrecan and growth factors, mechanical stimulation and oxygen tension will prove helpful to better chondrogenesis and to one day apply HUCMSCs not only to fibrocartilage but also hyaline cartilage tissue engineering [2].

\subsection{Clinical Trials}

Although there has been an increasing number of groups around the world (especially US) that are studying the use of HUCMSCs in cartilage repair, the clinical trials to date regarding the use of umbilical stem cells in cartilage repair have been few [23], however an ongoing clinical trial is evaluating the use of a newly developed treatment CARTISTEM ${ }^{\circledR}$ [23]. A combination of sodium hyaluronate and HUMSCs, this therapeutic agent is intended for single-dose use, aimed at cartilage regeneration in humans and restoration of cartilage defects especially concerning the knee as a result of degenerative diseases, trauma and aging [24].

\section{Bone: Osteogenic Differentiation and Possible Treatment of Osteoporosis in Future}

\subsection{Current Bone Treatment and Mesenchymal Stem Cells}

Bone, contrary to cartilage, has a very good blood supply with an intrinsic self-repair and restoring ability. Current methods for bone repair and treatment revolve around autologous bone grafts, using the patient's own bone. However this method is associated with certain problems including, infection, malformation and varying degrees of donor site morbidity. Decent treatment for certain grave bone injuries are yet to be developed. Being able to be integrated directly into biomaterials with optional in vitro cultivation before implantation with minimal side effects, bone differentiating mesenchymal stem cells have proved ideal for bone regeneration [25].

\subsection{Bone Differentiating Abilities of HUCMSCs}

Osteogenic differentiation of HUCMSCs has been triggered by treatment mainly with $\beta$-glycerophosphate and dexamethasone [18]. Along the duration of osteogenic differentiation the activation of several key player genes were noted. The activation of both Runx2, a main regulatory gene of osteogenesis, and TAZ, its transcriptional co-activator for osteocalcin expression, were noted [17]. Several studies showed the up-regulation of both osteopontin and osteocalcin genes [17] [18]. Throughout the differentiation, positive alkaline phosphatase (ALP), a phosphatase usually expressed in high quantities in bone, activity was also noted in certain studies. The formation of certain bone-specific immunostaining proteins such as osteocalcin, bone sialoprotein-2 and osteonectin were prominent. Alizarin red staining and Kossa staining led to the confirmation of mineralisation [2]. 


\subsection{BMSCs Compared to HUCMSCs in Bone Differentiation and Formation}

Similarly to HBMSCs, after 2 weeks, mineralisation was observed and the quality of gene expression shown by these two MSCs was similar in nature along this time period. However, unlike in chondrogenic studies, HBMSCs were shown to outcompete most HUCMSCs in bone formation under certain conditions. Von Kossa and alizarin staining revealed that HUCMSCs induced less mineralization when it came to larger bone nodules compared to HBMSCs [2]. Even after exposure to dihydroxyvitamin (D3) for the duration of 6 weeks, little osteogenesis was shown by the HUCMSCs, with minimal mineralisation and few ALP-positive cells [26].

Contrary to other inducing agents, HUCMSCs and BMSCs had similar expression of osteogenic phenotypes when differentiation was induced by bone morphogenic protein-2, BMP-2, also similar transduction pathways were activated in both. HUCMSCs were demonstrated to undergo osteogenic differentiation and proliferation when cultured on a calcium phosphate cement. Umbilical stem cells'osteogenic proliferation was inhibited, when cultured with a demineralized bone matrix medium, upon examination the stem cells looked flattened and shortened, while ALP activity increased substantially [2].

Although long lasting cultures will be needed in future to prove the residence of late stage osteogenic markers, the research mentioned above confirms that HUCMSCs may progress along the oestogenic lineage.

\subsection{Monolayer Cultures in 3D Biomaterials}

After exposure to osteoinductive biomaterials and chemical signals, monolayer cultures of HUCMSCs, showed signs of osteogenic differentiation with the evidence of mineralization and upregulation of osteonectin and Runx2 when cultured in 3D biomaterials. In polycaprolactone-tricalcium-phosphate scaffolds HBMSCs were found to demonstrate increased osteogenic activity with a significantly larger mineral percentage production when compared to that produced by HUCMSCs [2] [27].

Through various studies, HUCMSCs have been shown to undergo osteogenic differentiation through various applied techniques and in vitro mediums, such as when: encapsulated in alginate microbeads, when grown on fast-resorbable calcium phosphate bone cement, when seeded on specially designed polycaprolactone-collagenhydroxyapatite nanofibrous scaffolds and when seeded into injectable calcium phosphate-alginate hydrogel paste [2].

\subsection{Seeding Density for 3D Biomaterial Cultures}

It was discovered that the seeding density required for optimum differentiation and growth was that of 25 million cells/ml or more. It was also noted that switching to an anabolic medium IGF-1 with poly (L-lactic acid) from an osteogenic medium containing dexamethasone and vitamin D3 was not favourable [27].

\subsection{Different Constructs for HUCMSC Differentiation and Bone Formation and in Vitro Studies in Mice}

In order to evaluate promotion of osteogenesis and chondrogenesis in different constructs HUCMSCs were seeded into poly(L-lactic acid) scaffolds for 3 weeks with either an osteogenic or chondrogenic medium. These constructs were then sutured together, in some cases placing a small coat of undifferentiated HUCMSCs separating them. The insertion of HUCMSCs between the constructs significantly bettered the merging of the two structures, evidenced by histology, although differentiation was [28].

In another attempt, microsphere-based cylindrical scaffolds were designed to release BMP-2 from one side and TGF- $\beta$ from the other side, transitioning in release from one side to the other gradually and continuously. While there was evidence of localised Safranin-O staining on the TGF- $\beta$ side of the cylindrical construct, showing localised chondrogenesis in a chondrosteal approach, as well as a deposition of calcium throughout, differentiation was again restricted [29].

In a particular study, a method was used were a nano-hydroxyapatite-collagen-poly (L-lactic acid) composite, an artificial bone scaffold material, was seeded with HUCMSCs followed by subcutaneous implantation into mice. Although osteoblasts were detected after 12 weeks, using transmission electron microscopy, unfortunately no further investigations were conducted to see if these cells were differentiated HUCMSCs or host mouse cells [30].

In summary, HUCMSCs share some similar HBMSC osteogenic differentiation pathways: the activation of the TAZ and Runx2 gene and the upregulation of osteopontin and osteocalcin, resulting in mineralization. How- 
ever, the in vitro bone differentiating abilities of HUCMSCs, using methods to date, appears inferior to HBMSCs.

\subsection{HUCPVCs: Bone Formation, Repair and Regeneration}

Perivascular cells (a subpopulation of umbilical stem cells attained from the Wharton's jelly and tissue surrounding the umbilical vessels), when exposed to osteogenic medium, were seen to form bone nodules after the duration of only 4 - 5 days suggesting better bone differentiation compared to other HUCMSCS [31].

Comparing the two mesenchymal stem cells, HUCMSCs and HUCPVCs (human umbilical cord perivascular cells), the cell types share similar phenotypes save for CD146+ expression (16.2\% in HUCMSCs and a larger $37.9 \%$ of perivascular cells) [32]. Von Kossa staining, although positive with both MSCs, was slightly weaker in other HUCMSCs when compared to perivascular cells on exposure to osteogenic medium [2].

A recent study was conducted in order to determine the viability of CD146+ HUCPVCs in vivo osteogenesis. CD146+ HUCPVCs were introduced to a 3D Gelfoam-alginate complex producing an ectopic bone formation model prior to their subcutaneous transplantation into the backs of selected SCID (severe combined immunodeficient) mice. After the duration of 6 weeks, X-ray imaging showed that the transplants had undergone ectopic mineralisation. While histological staining of transplant sections uncovered osteocytes embedded in newly formed matrix, human specific mitochondria immunostaining indicated that the osteocytes nested in the bone matrix were of donor (HUCPVC) origin [32].

In order to assess the functional repair and regenerating abilities of the stem cells, a critical-sized bone defect was intentionally produced in the femur of a selected group of SCID, transplanting the 3D CD146+ HUCPVCsGelfoam-alginate complexes inside the damaged bone site. Newly formed bone was detected in the defect region on x-ray examination after a duration of 6 weeks after transplantation of CD146+ HUCPVCs transplantation similar with the previous model involving subcutaneous introduction of the stem cells. Apart from bone, a cartilaginous callus was also formed at the site of injury. Using particular techniques of mitochondria staining it was determined that not only were the CD146+ HUCPVCs incorporated within the new bone regenerate but they were also responsible for the regeneration and repair of the bone defect [33].

\subsection{Promising Finds in Future Treatment of Osteoporosis}

In a particular study, human umbilical cord blood -derived CD34+ cells were induced to undergo osteogenic differentiation and produce bone matrix under the stimulation of osteogenic signals, b-glycerophosphate and ascorbic acid. During this study several mice were treated with dexamethasone to bring about a change in bone microarchitecture and overall bone loss similar to that brought on by osteoporosis due to disproportionate increase in osteoclasts compared to osteoblasts also inhibiting osteoblastic action. Apart from obliterating bonemicroarchitecturel, osteoporosis also tends to direct bone marrow differentiation to adipogenesis [34].

After 28 days following application of the osteoporotic mice with the nanofiber-expanded CD34+ cells, the researchers observed a significant increase in osteoblastic activity increasing bone formation and a parallel decrease in the number of osteoclasts and adipocytes in osteoporotic sites. Apart from having an osteogenic effect on precursor cells, the elevated levels of CXCR4, chemokine receptor, expressed on the nanofiber CD34+ expanded cells were found to be indispensable in directing these CD34+ cells to bone marrow through their binding to stromal derived factor (SDF)-1 [34].

Elevated levels of granulocyte macrophage- colony stimulating factor (GM-CSF) secretion further evidenced the activity of osteoblastic action while the up-regulation of interleukin -10 (IL-10), a cytokine with anti-osteoclastic activity, evidenced the bone remodeling regulating abilities of the CD34+ in impairing osteoclastogenesis and improving bone formation [34].

Therefore current findings regarding human cord blood-derived CD34+ cell transplantation have shown the ability of these cells to increase trabecular bone formation with an increased trabecular quantity and thickness and decreased expression of adipocytes in bone marrow in osteoporosis and thus might prove instrumental in future treatment of human osteoporosis. UC derived CD34+ cells have another additional advantage in that experimental model systems have not shown signs of oncogenic and transformation [34].

\subsection{Clinical Trials}

Few clinical trials have ever been carried out in the use of HUCMSCs for bone regeneration. However, one was 
recently conducted to explore the benefits of transplanting umbilical cord mesenchymal stem cells and bone marrow stem cells together in the possible treatment of avascular necrosis of the femoral head (ANFH). Thirty ANFH patients took part in the study (6 females and 24 males: 49 hips). Using the Association Research Circulation Osseous system, 25 of the hips were in phase III while 24 where in phase II [35].

Bone destruction improved in $89.7 \%$ hips (44/49) with a major improvement of bone deposition on the damaged femoral head, refer to Figure 2. Four of the phase III hips actually improved to phase II while the twenty four phase II hips, which also seemed to have improved by imaging, stayed in the same phase. Not only did this trial prove the efficacy of HUCMSCs and BMSCs in osteogenesis and repair, but the stem cells also lead to the restoration and revascularisation of necrotic femoral head as well as gradually improving other symptoms of claudication and pain [35].

In future human clinical trials should be done to analyse the sole use of umbilical stem cells in repair of damaged bone to better understand and analyse their therapeutic effect [35].

\section{Neurogenerative and Neuroprotective Abilities of Umbilical Stem Cells}

\subsection{Mesenchymal Stem Cells and Umbilical Cells in CNS injury and Neurodegenerative Diseases}

Mesenchymal stem cells have proved to be promising in neurogenesis, repair of brain and spinal cord injury repair. Intravenous autologous MSC transplantation conducted in patients suffering from spinal cord injury or chronic ischemic stroke, during phase 1 clinical trials, contributed to decreased long-term disability in the patients undergoing the trial, while patient follow-ups showed and caused no severe adverse consequences related to MSC transplantation. What is more is that recent studies and evidence have pointed towards MSC-based therapy not only enhancing neurogenesis in brain injury but also increasing the blood supply going towards neurones and glial through angiogenesis [7].

In recent years HUCMSCs and Wharton jelly MSCs (WJ-MSCs), obtained from umbilical Wharton jelly,

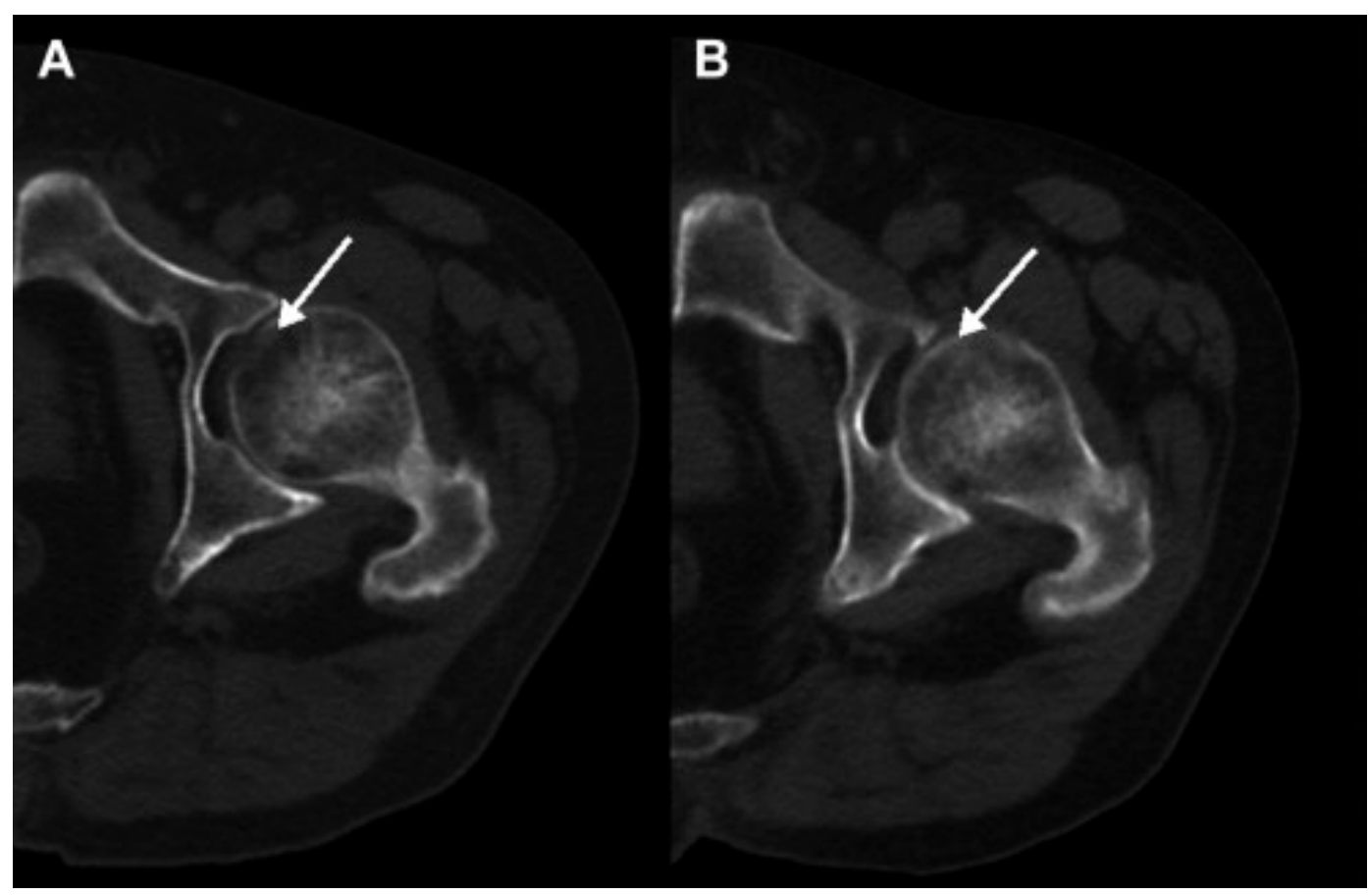

Figure 2. New bone regeneration and restoration was observed in patient's femoral head under imaging techniques. Left side of the figure above shows the necrosis at one patient's left femoral head before undergoing treatment, the upper-inner segment of the femoral head showing signs of capsular transparency (indicated by the white arrow on the left hand side image) in the inner-upper region of femoral head. However, after 1 year of treatment the once low-density region was restored with deposition of new bone tissue (as indicated by the white arrow on the right). Adapted from [35]. 
have had various breakthroughs in neural in vitro differentiation into cholinergic [15] and gabaergic [36] neurones as well as had positive outcomes in hippocampal neurone regeneration [37].

\subsection{Evidence on Neuronal Differentiating Abilities}

In a study carried out [38], Wharton's jelly cells were observed to go through morphological changes also expressing neural-specific proteins on induction by an adapted method from previous studies [39]. This study was one of the first in demonstrating the ability of these easily attainable multipotent stem cells to differentiate into neural cells in vitro. In both untreated and treated Wharton's jelly cells, neurone specific enolase (NSE), a neural stem cell marker, was found at approximately equal levels [38] homogenous with results obtained from bone marrow stromal cells [39]. Apart from NSE, cultivations of Wharton's jelly cells were also found to express ckit and $\mathrm{TH}$, the latter being a marker for catecholaminergic neurons [38].

During studies carried out by [40] after 3 days of being exposed to a neuronal conditioned medium, HUCMSCs started to exhibit and produce neuron-specific proteins such as neurofilament (NF) and NeuN. Past the $3^{\text {rd }}$ day various other changes were noted such as the development and elaboration of processes, rescission of the cell body and grouping of cells. Within a week the stem cells had started to express functional mRNA accountable for the synthesis of subunits of both glutamate decarboxylase and kainate receptors both essential to neuronal function and communication. Within less than two weeks, $87 \%$ of HUCMSCs were expressing NF and glutamate, the latter invoking an inward current that confirmed the stem cells' complete differentiation into mature neurones and achievement of neuronal functionality [40].

\subsection{Umbilical Stem Cells and Role of Beta1-Integrin in Treatment of Stroke}

HUCMSCs have been found useful in the treatment of stroke in rat models. Transplanted HUCMSCs were observed to differentiate into neuronal and glial cells as well as CXCR4+, doublecortin+, and vascular endothelial cells upon migration towards the ischeamic boundary zone, enhancing neuroplasticity in the ischemic brain [41].

Increased beta1-integrin expression was found to significally increase expression of neurotrophic factors. On the other hand inhibition of beta1-integrin expression not only reduced the ischaemic recovery from neurological deficit but inhibited local angiogenesis. Therefore regulation of beta1-integrin expression was also found to play a role in neuronal differentiation and plasticity of HUCMSCs particularly in the treatment of the ischemic brain [41].

\subsection{Secretome of Umbilical Stem Cells}

Transplanted MSCs such as Wharton's Jelly mesenchymal stem cells (WJMSCs) and HUCMSCs not only have the capability of directly differentiating into neurons after injection, but also secrete a MSC secretome which has an extensive range of immunomodulatory and trophic cytokines that have a substantial potential for the treatment of brain damage via stimulation of endogenous neurogenesis and neuro-protection. Resulting from the limited control and knowledge of cytokine production succeeding MSC transplantation, harvesting secretome for relevant therapeutic outcomes is still proving challenging [7]. The secretome and the MSCs recovered from Wharton jelly, have shown particular positive impacts on cell density and metabolic viability of primary hippocampal neurons when compared to MSC derived from adipose [37]. However when it comes to neuronal survival, factors enhancing effects of the WJ-MSC treated media are yet to be discovered.

\subsection{Neurogenerative Abilities of Umbilical Stem Cells Compared to Those of Bone Marrow Mesenchymal Stem Cells}

In a study conducted by [7], comparing collected BMSCs and WJ-MSCs, the DAVID (Database for Annotation, Visualisation and Integrated Discovery) web-based tool was applied to gene signatures in order to understand better the functional consequences of differing gene expression patterns. Using this GO (gene ontology) database system, it was demonstrated that the predominantly overrepresented biological processes in WJ-MSC involved those related to the development of the nervous system through neurogenesis and cell proliferation. Contrasting with this outcome, BM-MSCs mainly overrepresented processes pertaining to ossification and the overall development of the skeletal system. Identified as neurogenesis transcriptional factors in previous studies, PITX1 and SOX11 were observed to be largely expressed in WJ-MSCs and these results were reconfirmed by 
reverse transcription polymerase chain reaction (RT-PCR). Receptors such as NRP2, FLT1 and CDH2, involved in neurogenesis and angiogenesis, were also more numerous in WJ-MSCs when compared to BMSCs [7].

\subsection{Secretome of BMCs and WJ-MSCs and Their Chemotaxic Abilities on Neuro-2a Cells}

Exploring the secrotome of the MSCs, WJ-MSCs were found to express EGF, MDK, NTF3 and other such neurogenic cytokines in high amounts. The MSCs differed in the up-regulation of specific genes, with BM-MSCs namely up-regulating PGF and ANGPT1 expression, and the WJ-MSC up-regulating namely CXCL5, HBEGF, CXCL2, FGF9, EGF, NTF3 and MDK. Enzyme-linked immunosorbent assay (ELISA), allowed for the monitoring of secreted proteins levels where CXCL5 was produced in significantly higher amounts in the case WJMSCs and their conditioned medium while the BM-MSC conditioned medium was found to contain higher amounts of secreted platelet growth factor (PGF) [7].

Keeping in mind this difference in up-regulated genes between the two stem cell types as well as the differing secretomes, with their own concoction of cytokines and growth factors, the two MSCs were investigated in order to analyse their chemotaxic influence on neural cells as well as their neural enhancing abilities. The BMSCs and WJ-MScs were co-cultured separately with mouse Neuro-2a (N2a)cells (cells capable of axonal outgrowth and neuron differentiation) in Transwell plates in order to observe the chemotaxic effects on neural cells due to the MSCs. Although N2a migration was prompted by both MSCs types, N2a cells were clearly seen to travel towards the WJ-MSCs more regularly and quickly [7].

\subsection{Neuronal Differentiating Abilities of WJ-MSCs and BMSCs Secretomes}

Examining the neuronal differentiation inducing abilities of the MSCs upon N2a, N2a cells were cultured separately with the conditioned media from WJ-MSCs and BM-MSCs respectively, collecting the total proteins produced after the duration of 4 days [7].

Increased NEFL (Neurofilament light polypeptide) and TUBB3 ( $\beta$-tubulin III) marker expression, originating from the WJ-MSC conditioned medium, evidenced the conclusion of better neural differentiation induction of the N2a cells. Increased neurite-positive, TUBB3-positive cells were also present upon cultivating the N2a cells with WJ-MSC compared to BM-MSC conditioned medium furtherly confirming WJ-MSCs capability in neural differentiation induction [7].

\subsection{WJ-MSC Secretome and Its Neuroprotective Effects on Injured by Oxygen-Glucose Deprived Primary Cortical Cells}

Comparing the neuroprotective efficacy of BMSCs and WJ-MSCs and their secreted factors in oxygen glucose deprevation (OGD), a lab designed ischaemic model of was used to substitute a real life ischaemic insult to the central nervous system, WJ-MSCs were found to secrete factors with better neuroprotective qualities than those secreted from BM-MSCs. Following OGD, both co-cultures with BM-MSCs and WJ-MSCs, showed a compelling reduction in apoptosis cell death compared to the control, however the WJ-MSC group demonstrated the lowest count in cell death and apoptosis rate [7].

While the number of branched points, neurite length and overall neuronal proliferation was increased the most in the WJ-MSCs, the quantity of astrocytes was decreased in the WJ-MSC group when compared to the BMSCs [7].

\section{Umbilical Stem Cells Use and Treatment in Cerebral Palsy}

\subsection{Cerebral Palsy: The Disease}

Cerebral palsy is one most readily identifiable consequence of perinatal brain injury. This condition may occur due to chronic or acute injury sustained following birth in the new born period or even before, during the birthing process or during the development of the foetus [6]. Cerebral palsy is characterised by problems in movement and posture as well as disturbances of perception, cognition, communication, sensation and may also manifest itself in seizures as well as behavioural changes. The aetiologies causing the perinatal brain insult leading to the manifestation of the condition are numerous with the main culprits being: cerebral dysgenesis, leukomalacia, intracranial haemorrhage and periventricular intrapartum asphyxia [42]. 


\subsection{Cerebral Palsy and Umbilical Stem Cells: Why Umbilical Stem Cells?}

Currently there are a number of undergoing clinical trials worldwide targeting the use of umbilical stem cells in treatment of cerebral palsy. A couple of trials have already had some positive outcomes e.g. [14], but it is still going to be a couple of years before the medical community can draw some proper trial-based evidence on the use of the umbilical stem cells in cerebral palsy [6].

Due to the neuroprotective and neurogenerative properties that umbilical stem cells show, mentioned in the previous chapter, they have been considered as having promising potential in treatment of cerebral palsy. Umbilical cord blood transplantation has been especially promising in treatment of hypoxic-ischemic brain injury which is one of the major causes of cerebral palsy [43]. The little trials that have been completed have not been made publically available as peer-reviewed reports. Although some trials have now been completed, there have little peer-reviewed reports of them published as yet [6].

\subsection{Case Study Treatment in 5-Year-0ld Girl with Cerebral Palsy}

In a case study a cerebral palsy suffering 5-year-old girl, was treated using the umbilical cord of her sibling. The patient presented with symptoms of severe dysarthria restraining her speech, ambulation with tumble and other symptoms that were associated with retardation of motor function during congenital growth and a disability in standing. Upon physical examination and assessment the child's limb muscular tension appeared normal however her fine motor skills were lacking in finesse and were overall quite bad. On a psychological level the child was quite stressed and apprehensive compared to other children her age [42].

Although she had no history of epilepsia or chorea and her limbs had a normal muscular tension, she was found to lack fine motor skills and measured 48/126 in terms of functional independence. What is so interesting and promising about this case is that although neurotrophic drugs and other treatment produced limited positive results treating the cerebral palsy with the umbilical cord of her sibling had very positive results [42].

After processing of the MSCs, $5-10 \times 10^{6}$ MSCs in a 20 ml solution were administered per treatment, with $2 \mathrm{ml}$ being delivered by subarachnoid injection via lumbar puncture and $18 \mathrm{~mL}$ were delivered intravenously. The girl was treated seven times along the course of a year and during this time the patient only had a singular episode of mild fever which cleared up in 24 hours and except for rehabilitation training no other medical treatment was administered [42].

Post treatment (28 months) the patient was seen to improve in various areas, most noticeable of which was the major decrease in ambulation with tumble. The patient also developed the ability to stand up without the help of anyone. Further improvements included an improved adjustment in comprehension and speech, enhanced immunity and overall elevated levels of physical strength. What is more is that the treatment had little to no adverse effects with multiple renal and liver function tests, other blood tests and chest X-rays showing normal parameters [42].

This lone case proved that in children, at least, HUCMSCs have the capacity to improve the gross motor dysfunction experienced in cerebral palsy. However investigations into larger scale trials and studies are still going to be crucial in determining the possible merit and adverse effects of using these MSCs for cerebral palsy treatment [42].

\subsection{Coupled Treatment of Cerebral Palsy with EPO and Umbilical Cord Blood}

In a recent study the use of umbilical stem cells together with recombinant human erythropoietin (rhEPO) in treatment of cerebral palsy in children was assessed in a double-blind placebo- controlled randomized trial involving 105 children. The pUCB group of children were the group that were treated with rhEPO potentiated UCB and also received rehabilitation. The second group (EPO group) received rehabilitation and rhEPO with placebo UCB. The Control group on the other hand received both placebos, of UCB and rhEPO, only receiving the rehabilitation part of the treatment [14].

The results of the study consistently showed that compared to the control and EPO group, superior therapeutic outcomes were observed in the pUCB group. Be it 1-month, 3 months or 6-months post-treatment the children improved in both cognitive and motor function. However the motor abilities of the children less than 36 months old, according to the GMFM scale, were best improved in the EPO group [14].

However, apart from this outcome, the rhEPO had little effect, suggesting that the positive results obtained 
from the pUCB group [14] were mainly down to the UCB, even though rhEPO was expected to have a larger therapeutic effect and is described to have neurotrophic abilities [44].

However, amidst these positive results there were some minor adverse effects, with the pUCB group experiencing higher rates of irritability and pneumonia as non-serious adverse effects of the treatment. On intravenous administration of the UCB treatment there was an immediate decline in observed blood oxygen saturation. However providing the patients with oxygen quickly corrected the defecit. No severe adverse effects were recorded and the one death that did occur during the trial was found to be unrelated to treatment itself but rather due to the pre-existing severity of the patient's hypoxic ischaemic encephalopathy [14].

Due to the fact that EPO was used to boost the neuroprotective efficacy of the UCB, it cannot be taken for granted that the outcome of this trial would have been the same without the EPO in the pUCB group. Further studies should and are currently being done to study the individual effect of UCB and to highlight the differences in efficacy between allogenic (coming from matched donor) and autologous (coming from own harvested umbilical cord) originating UCB and to further enhance the positive effects of UCB on cerebral palsy. However when taking into consideration the severity and frequency of the adverse effects encountered during this study, the risks fall short of the overall benefits that lie in considering this new treatment for cerebral palsy [14].

\section{Promising Finds in Umbilical Stem Cell Treatment of Alzheimer's and Under- going Clinical Trials}

\subsection{Alzheimer's: the Disease}

Like other neurodegenerative disorders, the umbilical cord (containing stem cells with neurogenerative and neuroprotective properties) is being looked into as a possible treatment source for Alzheimer's disease (AD). This neurodegenerative disorder, which has also been described as being progressive and age-related, manifests itself in symptoms of serious cognitive decline and memory loss. The pathology of the disease is dominated by neuronal loss, deposition of amyloid plaques and neurofibrillary tangles. Having said this, however, increased deposition of amyloid $\beta$-peptide (A $\beta$ ) deposition is the main reason for the loss of neurones in AD [45]. The decrease in cognitive function comes from the damage and loss of cholinergic neurones innervating vast areas of cerebral cortex in the forebrain [15].

Therefore in vitro and vivo evidence of WJ-MSCs', derived from umbilical cord, ability to replace these cholinergic neurones through neuronal differentiation as well as reduce the deposition of the destructive amyloid protein has given life to the idea of using these umbilical stem cells for the treatment of Alzheimer's [15] [45].

\subsection{Differentiation into Cholinergic Neurons}

An in vitro study, conducted recently [15], it was observed that under the influence of fibroblast growth factor 8 (FGF8) as well as bone morphogenic protein 4 (BMP4), both known for their neural induction abilities [45] [46], Nscm treated WJ-MSCs were found to proliferate towards a cholinergic lineage. Their differentiation was confirmed visually through microscopic techniques as well as testing for the production of NF and the upregulation of choline acetyltransferase (CHAT) gene for the enzyme required for the production of acetylcholine, the neurotransmitter of cholinergic neurones. These results substantiate promising arguments in WJ-MSCs being for AD therapy and a source of cholinergic neurones [15].

\subsection{Stem Cell Ability to Reduce Levels of Amyloid $\beta$-Peptide Deposition in A $\beta$ PP/PS1 Transgenic Mice}

In another study conducted on A/PP/PS1 transgenic mouse model, tricyclodecan-9-yl-xanthogenate (D609) induced HUMSCs differentiated into neuron-like cells (HUMSC-NCs), were transplanted into the transgenic AD mouse model. The transplantation was found to improve the mice's cognitive function, reduce substantially the $\mathrm{A} \beta$ deposition in the mice together with the increase in production of synapsin 1 [45].

The cause of these beneficial effects was found to be due to the increase in M2-like microglial activation in those transplanted with the HUMSC-NCs, increasing in turn the expression of interleukin-4 (IL-4), a M2-like microglia associated anti-inflammatory cytokine. Tumour necrosis factor $\alpha$ (TNF- $\alpha$ ) as well as interleukin- $1 \beta$ (IL-1 $\beta$ ), both cytokines of a pro-inflammatory nature, typically of M1-like microglial expression were substantially decreased. What is more is that the expression of neprilysin (NEP) and insulin-degrading enzyme (IDE), 
both $\mathrm{A} \beta$ degrading entities, were considerably increased in those mice treated with stem cells [45].

All these results point to the ability of HUMSC-NC transplantation to decrease the deposition of amylin proteins as well as decrease neuroinflamation, both typically seen in Alzheimer's. Although these results were seen in $\mathrm{A} \beta \mathrm{PP} / \mathrm{PS} 1$ transgenic mice and not in man, this information testifies to the promising cell therapy that umbilical stem cells can offer to the Alzheimer's community with future testing and clinical trials [45].

\subsection{Clinical Trials}

Unfortunately little known clinical trials have been conducted regarding the use of umbilical stem cells in treatment of Alzheimer's' disease and the true potential of this stem cell treatment can't be realised until more time and money is invested in human based treatment.

There is, however, a current ongoing trial that started in 2012 aimed at investigating the tolerability and safety of HUCMSCs and the effectiveness of their use in treatment of AD patients [47].

\section{Conclusions}

In conclusion, it is clear that umbilical stem cells have a large therapeutic potential, having a great advantage over other stem cells such as bone marrow and embryological stem cells due to fact that their use comes with little ethical issues.

This review has shed light on a few of the many uses of these stem cells such as their use in cartilage and bone formation and regeneration. This review also included evidence-based information on the stem cells' neurogenerative abilities and the neuroprotective effects of their secretome leading to them being used and studied for their therapeutic role in neurodegenerative diseases such as Cerebral palsy and Alzheimer's. HUMSCs were also pointed out to be having more efficient and powerful cartilage and neuronal differentiating abilities when compared to bone marrow stem cells, however, studies showed that BMSCs have better bone differentiating abilities than HUMSCs.

The actual use of umbilical stem cells in human clinical trials is still relatively a young concept but is luckily growing in popularity with many clinical trials currently underway worldwide assessing the stem cells' use in multitudes of conditions. There have been a few of these trials that have been published already as mentioned in this review. However, many more results are yet to be published and trials themselves are yet to be completed.

So with the promise of ongoing clinical trials, the next couple of years will be more fruitful in revealing information about the use of these stem cells in humans. However, the preliminary in vitro and in vivo experiments that have been conducted, as well as the human trial results that have been published so far, all point towards umbilical stem cells having great potential in various areas of medicine.

\section{Acknowledgements}

I would like to thank and acknowledge Chev. Dr. Blundell for his constant guidance during the course of this review. He helped mould and fine tune my ideas as well as suggest possible adjustments to make this review even better.

\section{References}

[1] Mennan, C., Wright, K., Bhattacharjee, A., Balain, B., Richardson, J. and Roberts, S. (2013) Isolation and Characterisation of Mesenchymal Stem Cells from Different Regions of the Human Umbilical Cord. BioMed Research International, 2013, Article ID: 916136. http://dx.doi.org/10.1155/2013/916136

[2] Wang, L.M., Ott, L., Seshareddy, K., Weiss, M.L. and Detamore, M.S. (2011) Musculoskeletal Tissue Engineering with Human Umbilical Cord Mesenchymal Stromal Cells. Regenerative Medicine, 6, 95-109. http://dx.doi.org/10.2217/rme.10.98

[3] Kakinuma, S., Tanaka, Y., Chinzei, R., Watanabe, M., Shimizu-Saito, K., Hara, Y., Teramoto, K., Arii, S., Sato, C., Takase, K., Yasumizu, T. and Teraoka, H. (2003) Human Umbilical Cord Blood as a Source of Transplantable Hepatic Progenitor Cells. Stem Cells, 21, 217-227. http://dx.doi.org/10.1634/stemcells.21-2-217

[4] Chao, Y.H., Wu, H.P., Chan, C.K., Tsai, C., Peng, C.T. and Wu, K.H. (2012) Umbilical Cord-Derived Mesenchymal Stem Cells for Hematopoietic Stem Cell Transplantation. Journal of Biomedicine and Biotechnology, 2012, Article ID: 759503. http://dx.doi.org/10.1155/2012/759503 
[5] Acosta, S.A., Franzese, N., Staples, M., Weinbren, N.L., Babilonia, M., Patel, J., Merchant, N., Simancas, A.J., Slakter, A., Caputo, M., Patel, M., Franyuti, G., Franzblau, M.H., Suarez, L., Gonzales-Portillo, C., Diamandis, T., et al. (2013) Human Umbilical Cord Blood for Transplantation Therapy in Myocardial Infarction. Journal of Stem Cell Research \& Therapy, S4, S4-005.

[6] Castillo-Melendez, M., Yawno, T., Jenkin, G. and Miller, S.L. (2013) Stem Cell Therapy to Protect and Repair the Developing Brain: A Review of Mechanisms of Action of Cord Blood and Amnion Epithelial Derived Cells. Frontiers in Neuroscience, 7, 194. http://dx.doi.org/10.3389/fnins.2013.00194

[7] Hsieh, J.Y., Wang, H.W., Chang, S.J., Liao, K.H., Lee, I.H., Lin, W.S., Wu, C.H., Lin, W.Y. and Cheng, S.M. (2013) Mesenchymal Stem Cells from Human Umbilical Cord Express Preferentially Secreted Factors Related to Neuroprotection, Neurogenesis, and Angiogenesis. PLoS One, 8, e72604. http://dx.doi.org/10.1371/journal.pone.0072604

[8] Lee, K.O., Gan, S.U. and Calne, R.Y. (2012) Stem Cell Therapy for Diabetes. Indian Journal of Endocrinology and Metabolism, 16, S227-S229.

[9] Shrestha, C., Zhao, L., Chen, K., He, H. and Mo, Z. (2013) Enhanced Healing of Diabetic Wounds by Subcutaneous Administration of Human Umbilical Cord Derived Stem Cells and Their Conditioned Media. International Journal of Endocrinology, 2013, Article ID: 592454. http://dx.doi.org/10.1155/2013/592454

[10] Chicha, L., Smith, T. and Guzman, R. (2014) Stem Cells for Brain Repair in Neonatal Hypoxia-Ischemia. Child's Nervous System, 30, 37-46. http://dx.doi.org/10.1007/s00381-013-2304-4

[11] Nikravesh, M.R., Jalali, M., Ghafaripoor, H.A., Sanchooli, J., Hamidi, D., Mohammadi, S. and Seghatoleslam, M. (2011) Therapeutic Potential of Umbilical Cord Blood Stem Cells on Brain Damage of a Model of Stroke. Journal of Cardiovascular and Thoracic Research, 3, 117-122.

[12] Lv, Y.T., Zhang, Y., Liu, M., Qiuwaxi, J.N., Ashwood, P., Cho, S.C., Huan, Y., Ge, R.C., Chen, X.W., Wang, Z.J., Kim, B.J. and Hu, X. (2013) Transplantation of Human Cord Blood Mononuclear Cells and Umbilical Cord-Derived Mesenchymal Stem Cells in Autism. Journal of Translational Medicine, 11, 196.

[13] Zhang, S., Chen, L., Liu, T., Zhang, B., Xiang, D., Wang, Z. and Wang, Y. (2012) Human Umbilical Cord Matrix Stem Cells Efficiently Rescue Acute Liver Failure through Paracrine Effects Rather than Hepatic Differentiation. Tissue Engineering Part A, 18, 1352-1364. http://dx.doi.org/10.1089/ten.tea.2011.0516

[14] Min, K., Song, J., Kang, J.Y., Ko, J., Ryu, J.S., Kang, M.S., Jang, S.J., Kim, S.H., Oh, D., Kim, M.K., Kim, S.S. and Kim, M. (2013) Umbilical Cord Blood Therapy Potentiated with Erythropoietin for Children with Cerebral Palsy: A Double-Blind, Randomized, Placebo-Controlled Trial. Stem Cells, 31, 581-591. http://dx.doi.org/10.1002/stem.1304

[15] Liang, J., Wu, S., Zhao, H., Li, S.L., Liu, Z.X., Wu, J. and Zhou, L. (2013) Human Umbilical Cord Mesenchymal Stem Cells Derived from Wharton's Jelly Differentiate into Cholinergic-Like Neurons in Vitro. Neuroscience Letters, 532, 59-63. http://dx.doi.org/10.1016/j.neulet.2012.11.014

[16] Wang, L. and Detamore, M.S. (2009) Insulin-Like Growth Factor-I Improves Chondrogenesis of Predifferentiated Human Umbilical Cord Mesenchymal Stromal Cells. Journal of Orthopaedic Research, 27, 1109-1115. http://dx.doi.org/10.1002/jor.20848

[17] Ciavarella, S., Dammacco, F., De Matteo, M., Loverro, G. and Silvestris, F. (2009) Umbilical Cord Mesenchymal Stem Cells: Role of Regulatory Genes in Their Differentiation to Osteoblasts. Stem Cells and Development, 18, 12111220. http://dx.doi.org/10.1089/scd.2008.0340

[18] Wang, H.S., Hung, S.C., Peng, S.T., Huang, C.C., Wei, H.M., Guo, Y.J., Fu, Y.S., Lai, M.C. and Chen, C.C. (2004) Mesenchymal Stem Cells in the Wharton's Jelly of the Human Umbilical Cord. Stem Cells, 22, 1330-1337. http://dx.doi.org/10.1634/stemcells.2004-0013

[19] Karahuseyinoglu, S., Cinar, O., Kilic, E., Kara, F., Akay, G.G., Demiralp, D.O., Tukun, A., Uckan, D. and Can, A. (2007) Biology of Stem Cells in Human Umbilical Cord Stroma: In Situ and in Vitro Surveys. Stem Cells, 25, 319-331. http://dx.doi.org/10.1634/stemcells.2006-0286

[20] Wang, L., Seshareddy, K., Weiss, M.L. and Detamore, M.S. (2009) Effect of Initial Seeding Density on Human Umbilical Cord Mesenchymal Stromal Cells for Fibrocartilage Tissue Engineering. Tissue Engineering Part A, 15, 10091017. http://dx.doi.org/10.1089/ten.tea.2008.0012

[21] Wang, L., Tran, I., Seshareddy, K., Weiss, M.L. and Detamore, M.S. (2009) A Comparison of Human Bone Marrow-Derived Mesenchymal Stem Cells and Human Umbilical Cord-Derived Mesenchymal Stromal Cells for Cartilage Tissue Engineering. Tissue Engineering Part A, 15, 2259-2266. http://dx.doi.org/10.1089/ten.tea.2008.0393

[22] Hellingman, C.A., Koevoet, W. and van Osch, G.J. (2012) Can One Generate Stable Hyaline Cartilage from Adult Mesenchymal Stem Cells? A Developmental Approach. Journal of Tissue Engineering and Regenerative Medicine, 6, e1-e11. http://dx.doi.org/10.1002/term.502

[23] Anderson, J.A., Little, D., Toth, A.P., Moorman III, C.T., Tucker, B.S., Ciccotti, M.G. and Guilak, F. (2014) Stem Cell Therapies for Knee Cartilage Repair: The Current Status of Preclinical and Clinical Studies. American Journal of 
Sports Medicine, 42, 2253-2261.

[24] Medipost Co Ltd. (2013) Evaluation of Safety and Exploratory Efficacy of CARTISTEM, a Cell Therapy Product for Articular Cartilage Defects.

[25] Salgado, A.J., Coutinho, O.P. and Reis, R.L. (2004) Bone Tissue Engineering: State of the Art and Future Trends. Macromolecular Bioscience, 4, 743-765. http://dx.doi.org/10.1002/mabi.200400026

[26] Majore, I., Moretti, P., Stahl, F., Hass, R. and Kasper, C. (2011) Growth and Differentiation Properties of Mesenchymal Stromal Cell Populations Derived from Whole Human Umbilical Cord. Stem Cell Reviews and Reports, 7, 17-31. http://dx.doi.org/10.1007/s12015-010-9165-y

[27] Wang, L., Singh, M., Bonewald, L.F. and Detamore, M.S. (2009) Signalling Strategies for Osteogenic Differentiation of Human Umbilical Cord Mesenchymal Stromal Cells for 3D Bone Tissue Engineering. Journal of Tissue Engineering and Regenerative Medicine, 3, 398-404. http://dx.doi.org/10.1002/term.176

[28] Wang, L., Zhao, L. and Detamore, M.S. (2011) Human Umbilical Cord Mesenchymal Stromal Cells in a Sandwich Approach for Osteochondral Tissue Engineering. Journal of Tissue Engineering and Regenerative Medicine, 5, 712721. http://dx.doi.org/10.1002/term.370

[29] Dormer, N.H., Singh, M., Wang, L., Berkland, C.J. and Detamore, M.S. (2010) Osteochondral Interface Tissue Engineering Using Macroscopic Gradients of Bioactive Signals. Annals of Biomedical Engineering, 38, 2167-2182. http://dx.doi.org/10.1007/s10439-010-0028-0

[30] Diao, Y., Ma, Q., Cui, F. and Zhong, Y. (2009) Human Umbilical Cord Mesenchymal Stem Cells: Osteogenesis in Vivo as Seed Cells for Bone Tissue Engineering. Journal of Biomedical Materials Research Part A, 91, 123-131. http://dx.doi.org/10.1002/jbm.a.32186

[31] Sarugaser, R., Lickorish, D., Baksh, D., Hosseini, M.M. and Davies, J.E. (2005) Human Umbilical Cord Perivascular (HUCPV) Cells: A Source of Mesenchymal Progenitors. Stem Cells, 23, 220-229. http://dx.doi.org/10.1634/stemcells.2004-0166

[32] Martin-Rendon, E., Sweeney, D., Lu, F., Girdlestone, J., Navarrete, C. and Watt, S.M. (2008) 5-Azacytidine-Treated Human Mesenchymal Stem/Progenitor Cells Derived from Umbilical Cord, Cord Blood and Bone Marrow Do Not Generate Cardiomyocytes in Vitro at High Frequencies. Vox Sanguinis, 95, 137-148. http://dx.doi.org/10.1111/j.1423-0410.2008.01076.X

[33] Tsang, W.P., Shu, Y., Kwok, P.L., Zhang, F., Lee, K.K., Tang, M.K., Li, G., Chan, K.M., Chan, W.Y. and Wan, C. (2013) CD146+ Human Umbilical Cord Perivascular Cells Maintain Stemness under Hypoxia and as a Cell Source for Skeletal Regeneration. PLoS ONE, 8, e76153. http://dx.doi.org/10.1371/journal.pone.0076153

[34] Aggarwal, R., Lu, J., Kanji, S., Joseph, M., Das, M., Noble, G.J., McMichael, B.K., Agarwal, S., Hart, R.T., Sun, Z., Lee, B.S., Rosol, T.J., Jackson, R., Mao, H.Q., Pompili, V.J. and Das, H. (2012) Human Umbilical Cord Blood-Derived CD34+ Cells Reverse Osteoporosis in NOD/SCID Mice by Altering Osteoblastic and Osteoclastic Activities.

[35] Cai, J., Wu, Z., Huang, L., Chen, J., Wu, C., Wang, S., Deng, Z., Wu, W., Luo, F. and Tan, J. (2014) Cotransplantation of Bone Marrow Mononuclear Cells and Umbilical Cord Mesenchymal Stem Cells in Avascular Necrosis of the Femoral Head. Transplantation Proceedings, 46, 151-155. http://dx.doi.org/10.1016/j.transproceed.2013.06.021

[36] Ali, H., Bayatti, N., Lindsay, S., Dashti, A.A. and Al-Mulla, F. (2013) Directed Differentiation of Umbilical Cord Blood Stem Cells into Cortical GABAergic Neurons. Acta Neurobiologiae Experimentalis, 73, 250-259.

[37] Ribeiro, C.A., Fraga, J.S., Graos, M., Neves, N.M., Reis, R.L., Gimble, J.M., Sousa, N. and Salgado, A.J. (2012) The Secretome of Stem Cells Isolated from the Adipose Tissue and Wharton Jelly Acts Differently on Central Nervous System Derived Cell Populations. Stem Cell Research \& Therapy, 3, 18. http://dx.doi.org/10.1186/scrt109

[38] Mitchell, K.E., Weiss, M.L., Mitchell, B.M., Martin, P., Davis, D., Morales, L., Helwig, B., Beerenstrauch, M., Abou-Easa, K., Hildreth, T., Troyer, D. and Medicetty, S. (2003) Matrix Cells from Wharton’s Jelly form Neurons and Glia. Stem Cells, 21, 50-60. http://dx.doi.org/10.1634/stemcells.21-1-50

[39] Woodbury, D., Schwarz, E.J., Prockop, D.J. and Black, I.B. (2000) Adult Rat and Human Bone Marrow Stromal Cells Differentiate into Neurons. Journal of Neuroscience Research, 61, 364-370. http://dx.doi.org/10.1002/1097-4547(20000815)61:4<364::AID-JNR2>3.0.CO;2-C

[40] Fu, Y.S., Shih, Y.T., Cheng, Y.C. and Min, M.Y. (2004) Transformation of Human Umbilical Mesenchymal Cells into Neurons in Vitro. Journal of Biomedical Science, 11, 652-660. http://dx.doi.org/10.1007/BF02256131

[41] Ding, D.C., Shyu, W.C., Chiang, M.F., Lin, S.Z., Chang, Y.C., Wang, H.J., Su, C.Y. and Li, H. (2007) Enhancement of Neuroplasticity through Upregulation of Beta1-Integrin in Human Umbilical Cord-Derived Stromal Cell Implanted Stroke Model. Neurobiology of Disease, 27, 339-353. http://dx.doi.org/10.1016/j.nbd.2007.06.010

[42] Wang, L., Ji, H., Zhou, J., Xie, J., Zhong, Z., Li, M., Bai, W., Li, N., Zhang, Z., Wang, X., Zhu, D., Liu, Y. and Wu, M. (2013) Therapeutic Potential of Umbilical Cord Mesenchymal Stromal Cells Transplantation for Cerebral Palsy: A Case Report. Case Reports in Transplantation, 2013, Article ID: 146347. http://dx.doi.org/10.1155/2013/146347 
[43] Wang, F., Maeda, N., Yasuhara, T., Kameda, M., Tsuru, E., Yamashita, T., Shen, Y., Tsuda, M., Date, I. and Sagara, Y. (2012) The Therapeutic Potential of Human Umbilical Cord Blood Transplantation for Neonatal Hypoxic-Ischemic Brain Injury and Ischemic Stroke. Acta Medica Okayama, 66, 429-434.

[44] Statler, P.A., McPherson, R.J., Bauer, L.A., Kellert, B.A. and Juul, S.E. (2007) Pharmacokinetics of High-Dose Recombinant Erythropoietin in Plasma and Brain of Neonatal Rats. Pediatric Research, 61, 671-675. http://dx.doi.org/10.1203/pdr.0b013e31805341dc

[45] Yang, H., Xie, Z., Wei, L., Yang, H., Yang, S., Zhu, Z., Wang, P., Zhao, C. and Bi, J. (2013) Human Umbilical Cord Mesenchymal Stem Cell-Derived Neuron-Like Cells Rescue Memory Deficits and Reduce Amyloid-Beta Deposition in an AbetaPP/PS1 Transgenic Mouse Model. Stem Cell Research \& Therapy, 4, 76. http://dx.doi.org/10.1186/scrt227

[46] Berse, B., Szczecinska, W., Lopez-Coviella, I., Madziar, B., Zemelko, V., Kaminski, R., Kozar, K., Lips, K.S., Pfeil, U. and Blusztajn, J.K. (2005) Expression of High Affinity Choline Transporter during Mouse Development in Vivo and Its Upregulation by NGF and BMP-4 in Vitro. Developmental Brain Research, 157, 132-140. http://dx.doi.org/10.1016/j.devbrainres.2005.03.013

[47] Affiliated Hospital to Academy of Military Medical Sciences (2012) Safety and Efficiency of Umbilical Cord-Derived Mesenchymal Stem Cells (UC-MSC) in Patients with Alzheimer's Disease (SEMAD). 


\section{Table of Abbreviations}

\begin{tabular}{|c|c|}
\hline Abbreviations & Meaning \\
\hline UC & Umbilical Cord \\
\hline MSC(s) & Mesenchymal Stem Cell(s) \\
\hline HUCMSC(s) & Human Umbilical Cord Mesenchymal Stem Cell(s) \\
\hline BMSC(s) & Bone Marrow Derived Mesenchymal Stem Cell(s) \\
\hline TGF & Tissue Growth Factor \\
\hline IGF-1 & Insulin Growth Factor-1 \\
\hline GAG(s) & Glycosaminoglycan(s) \\
\hline TMJ & Temporomandibular Joint \\
\hline D3 & Dihydroxyvitamin \\
\hline ALP & Alkaline Phosphatase \\
\hline BMP-2 & Bone Morphogenic Protein-2 \\
\hline SCID & Severe Combined Immunodeficiency/Deficient \\
\hline HUCPVC(s) & Human Umbilical Cord Perivacular Cell(s) \\
\hline SDF & Stromal Derived Factor \\
\hline GM-CSF & Granulocyte Macrophage-colony Stimulating Factor \\
\hline ANFH & Avascular necrosis of Femoral Head \\
\hline WJ-MSC(s) & Wharton Jelly-Mesenchymal Stem Cell(s) \\
\hline NSE & Neurone Spefic Enolase \\
\hline NF & Neurofilament \\
\hline DAVID & Database for Annotation, Visualisation and Integrated Discovery \\
\hline GO & Gene Ontology \\
\hline RT-PCR & Reverse Transcriptio-Polymerase Chain Reaction \\
\hline ELISA & Enzyme Linked Immunosorbent Array \\
\hline PGF & Platelet Growth Factor \\
\hline N2a & Neuro-2a \\
\hline NEFL & Neurofilament Light polypeptide \\
\hline TUBB3 & $\beta$ Tubulin III \\
\hline OGD & Oxygen Glucose Deprivation \\
\hline rhEPO & Recombinant Human Erythropoietin \\
\hline UCB & Umbilical cord Blood \\
\hline $\mathrm{A} \beta$ & Amyloid $\beta$ \\
\hline FGF8 & Fibroblast Growth Factor \\
\hline BMP-4 & Bone Morphogenic Protein-4 \\
\hline CHAT & Choline Acetyltransferase \\
\hline IL-4 & Interleukin-4 \\
\hline $\mathrm{TNF} \alpha$ & Tumour Necrosis Factor $\alpha$ \\
\hline $\mathrm{IL}-1 \beta$ & Interleukin- $1 \beta$ \\
\hline NEP & Neprilysin \\
\hline IDE & Insulin Degrading Enzyme \\
\hline
\end{tabular}

